\title{
Oral Findings and Salivary Parameters in Children with Celiac Disease: A Preliminary Study
}

\author{
Sibel Acar ${ }^{\mathrm{a}}$ Arzu Aykut Yetkıner ${ }^{\mathrm{a}}$ Nazan Ersın ${ }^{\mathrm{a}}$ Ozant Oncag ${ }^{\mathrm{a}}$ \\ Sema Aydogdu ${ }^{b}$ Cigdem Arıkan ${ }^{b}$
}

Departments of a Pediatric Dentistry and ${ }^{b}$ Pediatric Gastroenterology, Hepatology and Nutrition, Ege University, Izmir, Turkey

\section{Key Words}

Celiac disease $\cdot$ Dental defects $\cdot$ Recurrent aphthous stomatitis $\cdot$ Mutans streptococci $\cdot$ Lactobacilli $\cdot$ Caries $\cdot$ Saliva

\begin{abstract}
Objective: The aim of this study was to investigate the prevalence of dental enamel defects, recurrent aphthous stomatitis (RAS) and caries experience and to measure salivary flow rate, buffer capacity, saliva and plaque $\mathrm{pH}$ and salivary cariogenic microflora in patients with celiac disease (CD) compared to healthy subjects. Subjects and Methods: Thirtyfive patients, aged 6-19 years, with a diagnosis of $C D$ and 35 healthy children of the same age participated in the study. Enamel defects were diagnosed and classified using Aine's classification. The patients with RAS and dental caries were recorded using WHO criteria. The parents were interviewed about various oral health-related factors. Saliva samples were collected to measure the stimulated salivary flow rate, buffer capacity and $\mathrm{pH}$ values of saliva and plaque. Salivary mutans streptococci and lactobacilli were counted. Results: The enamel defects and RAS prevalence were statistically higher ( 40 and $37.1 \%$, respectively) in the CD group, and the prevalence of salivary mutans streptococci (48 and 14\%) and
\end{abstract}

lactobacilli (51 and 34\%) colonization was statistically lower $(p=0.012, p=0.010)$ in the CD group; the DMFS and dfs values were similar in both groups. Conclusion: $C D$ appeared to be associated with a significantly higher prevalence of developing enamel defects and RAS, but a lower prevalence of salivary mutans streptococci and lactobacilli colonization, and the diagnosis of these oral manifestations might be helpful for an early diagnosis of CD.

Copyright $\odot 2011$ S. Karger AG, Basel

\section{Introduction}

Celiac disease (CD) is defined as immunological responsiveness to ingested gluten in genetically susceptible individuals. It is characterized by a lifelong intolerance to the gluten contained in wheat, rye and barley. Chronic diarrhea, tiredness, abdominal distension and bloating, vomiting, weight loss, muscle weakness and loose stools are the characteristic clinical features [1]. In undiagnosed patients, it can cause serious developmental problems in childhood such as short stature and retardation of physical development and in adults potentially debilitating gastrointestinal symptoms seriously affecting quality of life and there is a possible association with depression and

\section{KARGER}

Fax +4161306 1234 E-Mail karger@karger.ch www.karger.com
(C) 2011 S. Karger AG, Basel

1011-7571/12/0212-0129\$38.00/0

Accessible online at:

www.karger.com/mpp
Sibel Acar

Department of Pediatric Dentistry

School of Dentistry, Ege University

TR-35100 Izmir (Turkey)

Tel. +90 232388 6431,E-Mail sibel_kibele@hotmail.com 
reduced bone mineral density. There is pathology of the mucous membrane of the small intestine, and the exact diagnosis can be verified by biopsy [2]. The only treatment of CD is strict and lifelong adherence to a glutenfree diet (GFD), which usually results in remission [3].

The prevalence of CD in Europe and the USA in children between 2.5 and 15 years of age is approximately 1:300 to 1:80 [4]. In Turkey, there are limited studies about the prevalence of $\mathrm{CD}$, but the prevalence in Turkish children ranges from 1 to $8.3 \%$ [5-7].

$\mathrm{CD}$ also presents oral manifestations, which are dental enamel defects and recurrent aphthous stomatitis (RAS) [3, 8-19]. The existence of an association between CD and dental enamel defects has been reported and the enamel defects have been attributed to hypocalcemia or a particular genetic condition that leads to a specific immune response to gluten [9].

Therefore, the objectives of this study were to investigate the prevalence of dental enamel defects, RAS, and caries experience, and to measure salivary flow rate, buffer capacity, saliva and plaque $\mathrm{pH}$, and salivary cariogenic microflora in $\mathrm{CD}$ and in healthy subjects.

\section{Subjects and Methods}

The study was approved by the Ethics Committee of Ege University, Bornova-Izmir, Turkey. Letters were sent to each individual or parent explaining the study to them. A total of 35 patients, 19 females and 16 males aged 6-19 years with a diagnosis of CD of 2 years' duration, confirmed by intestinal biopsy, were recruited for the study. Patients with CD had regular appointments at the Department of Pediatric Gastroenterology, Hepatology and Nutrition, Ege University and their response to GFD was checked at each appointment by a gastroenterologist. The same examiner (S.A.) examined all 35 patients. Another 35 subjects of the same age and social background who attended the Well Child Care Outpatient Clinic at Ege University, Faculty of Medicine for their regular checkup were recruited to a control group.

A questionnaire exploring additional systemic disease, medication, use of fluoride, oral hygiene habits, diet and the frequency of dental appointments was completed by all participants or their parents. The oral examination was carried out with a mouth mirror and probe under artificial light in the clinic using World Health Organization criteria (dfs and DMFS indices) [20]. The dental enamel defects were recorded according to Aine's classification [21]. Dental examination was performed by 1 examiner (S.A.). Before the examination of enamel defects and caries, the teeth were brushed with a prophylaxis paste, then washed, dried and the affected permanent teeth were observed.

The soft tissues were examined for the diagnosis of RAS and patients also were questioned about the recurrence of oral ulcers by the same examiner.

The analysis of saliva included the stimulated salivary flow rate, buffer capacity, saliva and plaque $\mathrm{pH}$, salivary mutans streptococci and lactobacilli counts. Participants chewed paraffin wax for $30 \mathrm{~s}$ for saliva stimulation based on the manufacturer's instructions (GC Corp., Japan) before saliva was collected. Salivary flow rate was recorded after a 5 -min collection period by using a Saliva Check Buffer (GC Corp.; $<3.5 \mathrm{ml}$ was very low, $3.5-5 \mathrm{ml}$ was low, $>5 \mathrm{ml}$ was normal). Buffering capacity was also recorded by using a Saliva Check Buffer. Using a pipette, sufficient saliva was drawn from the collection cup and dropped onto the test pads. Buffering capacity was grouped according to the change in colour of the test pads supplied by the manufacturer ( $0-5$ points: very low, $6-9$ points: low, and $10-12$ points: normal/high). The measurement of salivary $\mathrm{pH}$ was carried out with a $\mathrm{pH}$ test strip placed into the saliva sample for $10 \mathrm{~s}$. The change in the colour of the strip was compared with the testing chart in the package.

Plaque $\mathrm{pH}$ was recorded using the Plaque Indicator Kit's (GC Corp.) scale. Plaque was harvested from interproximal sites in the molar area by using the disposable plaque collection instrument. The plaque indicator solution was dispensed into the disposable dish and the instrument with attached plaque was dipped into the dish for $1 \mathrm{~s}$. The surface of the plaque turned green. Then the sample was left to ferment for $5 \mathrm{~min}$. The plaque samples changed color over time. The greatest acid production occurred at $5 \mathrm{~min}$, thus the $\mathrm{pH}$ value was measured over $5 \mathrm{~min}$ by checking the color and comparing it with the chart on the dispensing dish.

Salivary mutans streptococci and lactobacilli were counted by CRT Bacteria (Ivoclar-Vivadent, Liechtenstein). After removing the agar carrier from the plastic test vial, a sodium bicarbonate $\left(\mathrm{NaHCO}_{3}\right)$ tablet was placed at the bottom of the vial. The protective foils were removed from the two agar surfaces and the collected saliva samples were transferred to the test vial by a pipette from the test kit and spread on both sides of plastic vial. The agar carrier was inserted back into the vial and the vial was closed. The test vial was placed upright in the incubator, and incubated at $37^{\circ} \mathrm{C}$ for $48 \mathrm{~h}$. After the removal of the vial from the incubator, the colonies of mutans streptococci and lactobacilli were recorded according to the model chart given by the manufacturer.

\section{Statistical Analysis}

Data were analyzed by SPSS version 17 for Windows (SPSS, Chicago, Ill., USA); $\chi^{2}$ test and Fisher's exact test were used to assess statistical differences between categorical variables. MannWhitney $U$ test was used to determine significant differences between celiac patients and the control groups.

\section{Results}

\section{Dental Enamel Defects}

Enamel defects were observed in $14(40 \%)$ patients with CD. Of these 14, 12 (85.7\%) had defects of grade 1, and 2 (14.3\%) patients of grade 2 (fig. 1), while 21 of the CD patients did not have any defect. None of the subjects in the healthy group had enamel defects. This finding showed that the dental enamel defects occurred significantly $(\mathrm{p}<0.05)$ more often in CD patients. 
Table 1. Mean saliva and plaque $\mathrm{pH}$ values, salivary flow rates and buffering capacity of saliva in children with CD compared to healthy controls

\begin{tabular}{|c|c|c|c|c|c|c|c|c|}
\hline & \multirow{2}{*}{$\begin{array}{l}\text { Saliva } \\
\mathrm{pH}\end{array}$} & \multirow{2}{*}{$\begin{array}{l}\text { Plaque } \\
\mathrm{pH}\end{array}$} & \multicolumn{3}{|c|}{ Salivary flow rate } & \multicolumn{3}{|c|}{ Buffering capacity } \\
\hline & & & very low & low & normal & very low & low & normal/high \\
\hline Celiac group & 7.5 & 6.2 & 7 & 16 & 12 & 1 & 20 & 14 \\
\hline Control group & 7.3 & 6.1 & 3 & 13 & 19 & 0 & 19 & 16 \\
\hline
\end{tabular}

\section{Recurrent Aphthous Stomatitis}

Thirteen (37.1\%) of the CD patients had aphthous ulcers compared to $4(11.4 \%)$ in the control group. RAS was observed to be statistically (0.012) higher in patients with CD $(\mathrm{p}=0.012$, table 1$)$.

\section{Caries Experience}

The mean DMFS $( \pm S D)$ and dfs $( \pm S D)$ values were $2.74 \pm 5.7$ and $2.60 \pm 5.3$, respectively, in patients with $\mathrm{CD}$ and in the control group. The differences were not statistically significant $\left(\mathrm{p}_{\mathrm{DMFS}}=0.903 ; \mathrm{p}_{\mathrm{dfs}}=0.689\right)$.

\section{Saliva Analysis}

The salivary $\mathrm{pH}$, plaque $\mathrm{pH}$, salivary flow rate and buffering capacity are given in table 1 . In the $\mathrm{CD}$ group, 12 children (34.3\%) were found to have normal stimulated salivary flow rate, whereas 19 children (54.3\%) had normal rate in the healthy group (table 1). Healthy children had normal buffering capacities and the difference was not statistically significant $(\mathrm{p}=0.494)$. Similarly, plaque $\mathrm{pH}$ was also similar in both groups (6.2 in the $\mathrm{CD}$ group and 6.1 in the healthy group).

\section{Prevalence of Salivary Mutans Streptococci and \\ Lactobacilli}

Salivary mutans streptococci were low $\left(<10^{5}\right.$ mutans streptococci CFU/ml) in $17(48.65 \%)$ of the 35 patients with $\mathrm{CD}$ and in $5(14 \%)$ children in the control group, and the difference was statistically significant ( $\mathrm{p}<0.05$, fig. 2 ). Eighteen (51.4\%) children with CD and 12 (34.3\%) controls had $<10^{5}$ lactobacillus CFU/ml in saliva (fig. 3).

\section{Oral Hygiene}

Use of fluoride and the frequency of dental appointments were the same for both groups. Visible plaque was observed in $25(71.4 \%) \mathrm{CD}$ patients and $18(51.4 \%)$ healthy children, and the difference was not statistically different $(\mathrm{p}=0.76)$.

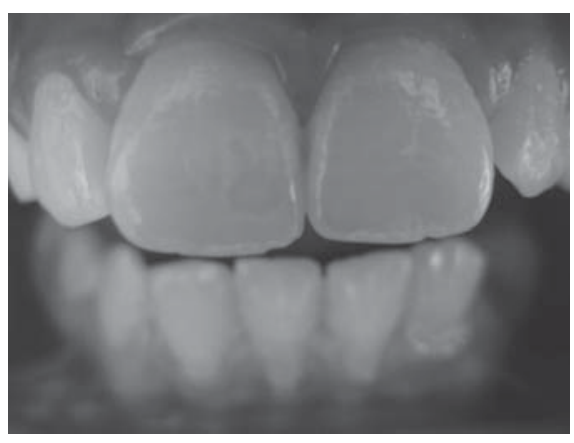

Fig. 1. CD patient with a grade 2 dental enamel defect on her permanent maxillary central incisors.

\section{Sugar Intake}

Based on CAT, no difference was found in the mean number of sugar exposures per day between $C D$ and healthy groups $(\mathrm{p}=0.125)$. For sugar intake, $19(54.3 \%)$ patients with CD and 16 (45.7\%) healthy children consumed sugar 1-2 times/day.

\section{Discussion}

The higher prevalence of specific enamel defects in CD patients than in controls in this study is similar to the findings of previous studies $[8,9,21,22]$. The etiology of dental enamel defects associated with CD is still uncertain. There are some hypotheses about the mechanism of enamel defects. One probable cause is hypocalcemia due to malabsorption during dental development because of low serum calcium concentration during enamel formation [23]. The malabsorption occurs due to intestinal damage in CD thereby leading to enamel hypoplasias resulting in hypocalcemia [24]. However, because some investigators $[9,11,25]$ did not find any statistically significant difference in the mean serum calcium concentra-

Med Princ Pract 2012;21:129-133 


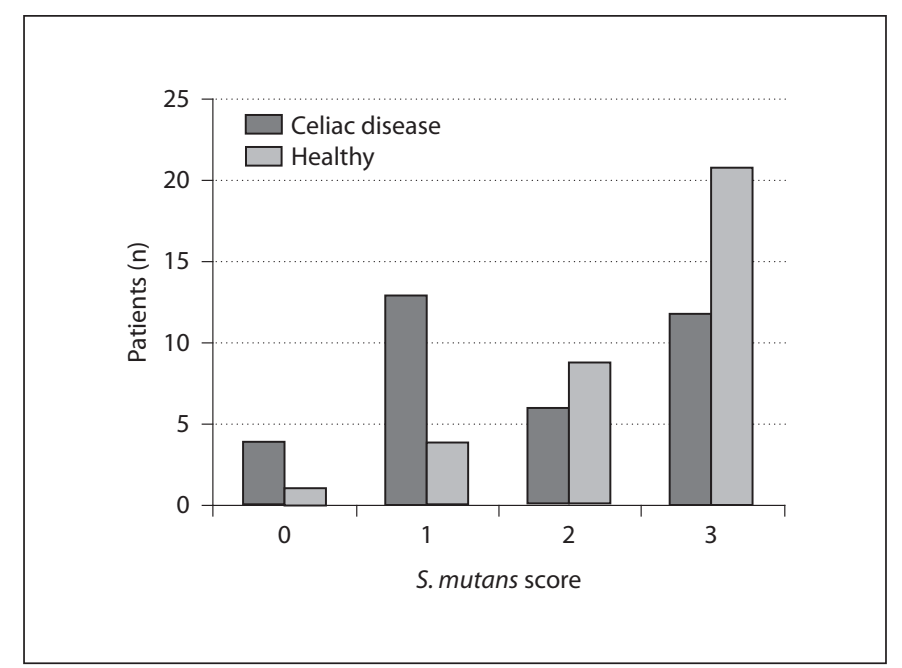

Fig. 2. Number of patients according to mutans streptococci scores in $\mathrm{CD}$ and healthy children.

tion between children with CD with and without enamel defects, they suggested that malabsorption could not be the cause of dental enamel defects $[9,11,25]$. In addition, Rasmusson and Eriksson [26] reported that CD did not have any significant influence on the mineralization of permanent teeth because the crowns of the deciduous and permanent teeth develop from 6 months to 7 years of life (except third molars), nutritional, immunological and genetic factors could be the possible cause of developmental defects in the enamel [25]. It has been reported that a specific antigen, human leukocyte antigen (HLA), alleles DR3 and DQ2, is strongly associated with dental defects in CD patients including their healthy first-degree relatives [10-12, 25].

The age at diagnosis of $\mathrm{CD}$ plays an important role in the occurrence of enamel defects. In our study, CD patients with dental enamel defects showed higher mean age of diagnosis of $\mathrm{CD}$ in comparison with the studies by Bucci et al. [8] and Hill et al. [4]. The mean age of diagnosis was 9.5 years in CD patients with enamel defects, and the CD patients without enamel defects had their diagnosis at the age of 7.8 years. Aguirre et al. [10] reported that early diagnosis of $\mathrm{CD}$ may account for the lower prevalence of enamel defects.

RAS was the other most common oral manifestation in our study, similar to previous reports $[14,15,17,19]$; its etiology is not known exactly. Genetic, immunological and microbial factors may play a role in the occurrence of RAS. Also local trauma, stress, food intake, some drugs,

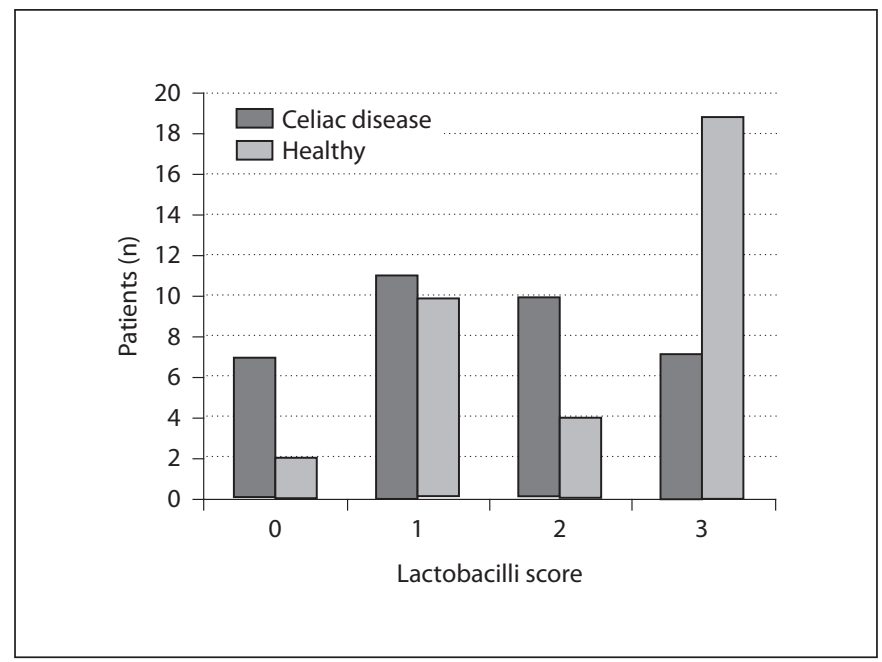

Fig. 3. Number of patients according to lactobacilli scores in CD and healthy children.

hormonal changes or vitamin and trace element deficiencies could play a role [27]. However, some studies reported nonsignificant differences in RAS in CD patients versus controls $[8,16]$.

The effect of GFD on the remission of RAS is still uncertain, but dietary withdrawal of gluten occasionally results in significant benefit. It was reported that CD patients with RAS showed a significant improvement in their aphthous lesions after GFD $[8,17,27]$. On the other hand, Hunter et al. [28] examined the effects of GFD in patients with RAS and without CD and found no statistical significance between the study group with GFD and the control groups. Our findings are in agreement with most studies. RAS was seen mostly in CD patients; it can be a risk indicator and an oral manifestation of $\mathrm{CD}$, but in our study we did not examine the effect of GFD on the remission of RAS.

In the present study the mean DMF and df index rates were similar in both groups to the values reported by other studies $[9,10,29]$. In addition, in some studies [10, 30], lower caries prevalence was reported in CD patients. It was suggested that this nonsignificant caries experience could be due to carefully controlled diets in patients with $\mathrm{CD}$, and thus they may have a low cariogenic diet and better dental care. Saliva parameters, including salivary flow rate, buffering capacity, saliva and plaque $\mathrm{pH}$, were also similar in both groups. However, the cariogenic microflora, levels of salivary mutans streptococci and lactobacilli, were found to be significantly lower in CD patients 
than in the healthy group, which could be due to their low cariogenic diets. Although no previous studies are available for comparison with our study, it could be suggested that the low cariogenic diet may affect the cariogenic microflora.

\section{Conclusion}

$\mathrm{CD}$ in children and young people was associated with a significantly higher prevalence of developmental enamel defects and RAS, and the diagnosis of these oral manifestations might be helpful for an early diagnosis of CD.

\section{References}

$\checkmark 1$ Leeds JS, Hopper AD, Sanders DS: Celiac disease. Br Med Bull 2008;88:157-170.

2 Jones R, Sleet S: Celiac disease. BMJ 2009;19: 338.

-3 Pastore L, Carroccio A, Compilato D, Panzarella V, Serpico R, Lo Muzio L: Oral manifestations of celiac disease. J Clin Gastroenterol 2008;42:224-232.

4 Hill ID, Dirks MH, Liptak GS, Colletti RB, Fasano A, Guandalini S, Hoffenberg EJ, Horvath K, Murray JA, Pivor M, Seidman EG, North American Society for Pediatric Gastroenterology, Hepatology and Nutrition: Guideline for the diagnosis and treatment of celiac disease in children: recommendations of the North American Society for Pediatric Gastroenterology, Hepatology and Nutrition. J Pediatr Gastroenterol Nutr 2005;40:1-19.

5 Gursoy S, Guven K, Simsek T, Yurci A, Torun E, Koc N, Patiroglu TE, Ozbakir O, Yucesoy $\mathrm{M}$ : The prevalence of unrecognized adult celiac disease in central Anatolia. J Clin Gastroenterol 2005;39:508-511.

-6 Altuntaş GB, Kansu A, Girgin N: Celiac disease in Turkish short-statured children and the value of antigliadin antibody in diagnosis. Acta Paediatr Jpn 1998;40:457-460.

-7 Tümer L, Hasanoğlu A, Aybay C: Endomysium antibodies in the diagnosis of celiac disease in short statured children with no gastrointestinal symptoms. Pediatr Int 2001; 43:71-73.

$\checkmark 8$ Bucci P, Carile F, Sangianantoni A, D’Angio F, Santarelli A, Lo Muzio L: Oral aphthous ulcers and dental enamel defects in children with coeliac disease. Acta Paediatr 2006;95: 203-207.

-9 Avsar A, Kalaycı AG: The presence and distribution of dental enamel defects and caries in children with celiac disease. Turk J Pediatr 2008;50:45-50.

10 Aguirre JM, Rodriguez R, Oribe D, Vitoria JC: Dental enamel defects in coeliac patients. Oral Surg Oral Med Oral Pathol Oral Radiol Endod 1997;84:646-650.
11 Mariani P, Mazzilli MC, Margutti G, Lionetti P, Triglione P, Petronzelli F, Ferrante E, Bonamico M: Coeliac disease, enamel defects and HLA typing. Acta Paediatr 1994;83: 1272-1275.

12 Ventura A, Martelossi S: Dental enamel defects and coeliac disease. Arch Dis Child 1997;77:91.

13 Paez EO, Lafuente PJ, Garcia PB, Lozano JM, Calvo JCL: Prevalence of dental enamel defects in celiac patients with deciduous dentition: a pilot study. Oral Surg Oral Med Oral Pathol Oral Radiol Endod 2008;106:74-78.

14 Procaccini M, Campisi G, Bufo P, Compilato D, Massaccesi C, Catassi C, Lo Muzio L: Lack of association between celiac disease and dental enamel hypoplasia in a case-control study from an Italian central region. Head Face Med 2007;3:25.

15 Campisi G, Di Liberto C, Carroccio A, Compilato D, Iacono G, Procaccini M, Di Fede G, Lo Muzio L, Craxi A, Catassi C, Scully C: Coeliac disease: oral ulcer prevalence, assessment of risk and association with glutenfree diet in children. Dig Liver Dis 2008;40: 104-107.

16 Sedghizadeh PP, Shuler CF, Allen CM, Beck FM, Kamlar JR: Celiac disease and recurrent aphthous stomatitis: a report and review of the literature. Oral Surg Oral Med Oral Pathol Oral Radiol Endod 2002;94:474-478.

17 Campisi G, Di Liberto C, Iacono G, Compilato D, Di Prima L, Calvino F, Di Marcos V, Lo Muzio L, Sferrazza C, Scalici C, Craxis A, Carroccio A: Oral pathology in untreated coeliac disease. Aliment Pharmacol Ther 2007;26:1529-1536.

18 Meini A, Pillan MN, Plebani A, Ugazio AG, Majorana A, Sapelli PL: High prevalence of DRW10 and DQW1 antigens in celiac disease associated with recurrent aphthous stomatitis. Am J Gastroenterol 1993;88:972.
19 Aydemir S, Tekin NS, Aktunc E, Numanoğlu G, Ustundag Y: Celiac disease in patients having recurrent aphthous stomatitis. Turk J Gastroenterol 2004;15:192-195.

20 World Health Organization: Oral Health Surveys: Basic Methods, ed 4. Geneva, WHO, 1997.

21 Aine L: Dental enamel defects and dental maturity in children and adolescents with celiac disease. Proc Finn Dent Soc 1986;82: $1-71$.

22 Wierink CD, van Diermen DE, Aartman IH, Heymans HS: Dental enamel defects in children with coeliac disease. Int J Paediatr Dent 2007;17:163-168.

23 Nikiforuk G, Fraser D: The etiology of enamel hypoplasia: a unifying concept. J Paediatr 1981;98:888-893.

24 Aine L, Mäki M, Collin P, Keyriläinen OJ: Dental enamel defects in celiac disease. Oral Pathol Med 1990;19:241-245.

25 Mäki M, Aine L, Lipsanen V, Koskimies S: Dental enamel defects in first-degree relatives of celiac patients. Lancet 1991;337:763-764.

26 Rasmusson CG, Eriksson MA: Coeliac disease and mineralisation disturbances of permanent teeth. Int J Paediatr Dent 2001;11: 179-183.

27 Shakeri R, Zamani F, Sotoudehmanesh R, Amiri A, Mohamadrejad M, Davarchi F, Karakani AM, Malekzadeh R, Shahram F: Gluten sensitivity enteropathy in patients with recurrent aphthous stomatitis. BMC Gastroenterol 2009;9:44.

28 Hunter IP, Ferguson MM, Scully C, Galloway AR, Main AN, Russell RI: Effects of dietary gluten elimination in patients with recurrent minor aphthous stomatitis and no detectable gluten enteropathy. Oral Surg Oral Med Oral Pathol 1993;75:595-598.

29 McLoughlin MJ, McNeill S: Incidence of dental caries in coeliac children. Arch Dis Child 1980;55:80-85.

30 Fulstow ED: Incidence of dental caries in coeliac children. Arch Dis Child1979;54:66167. 